\title{
ANCRAGE PERCEPTIF ET INVARIANT DANS LES LANGUES DES SIGNES (LS), LANGUES DE SOURDS
}

\author{
Brigitte GARCIA \\ Emmanuella MARTINOD \\ UMR 7023 Structures Formelles du Langage, Université Paris 8-Université Paris \\ Lumières et CNRS
}

\begin{abstract}
En): Sign languages (SLs), the visual-gestural languages used by deaf people throughout the world, display a higher degree of similarities than the one existing among spoken languages (oral-aural languages). The paper explores the hypothesis according to which this substantial invariant would be linked to the common rooting of SLs in a figurative depiction (iconicization) of human perceptive-practical experience. This issue is addressed through the study of a specific aspect: the comparison of the so-called morphemic handshapes throughout a panel of SLs. The study highlights the existence of a cross-SLs core of morphemic handshapes and shows that they can be analyzed as iconicization of percepts. This thereby supports the initial hypothesis and offers insight into the original input of SLs concerning the broad issue of the links between perception and language.
\end{abstract}

Keywords (En): sign language; percept; iconicization; invariant and variation; morphemic handshape; sign language typology

Mots-clés (Fr) : langues des signes ; percept ; iconicisation ; invariant et variation ; configuration morphémique; typologie des langues des signes

\section{Introduction}

Les langues des signes (LS), langues visuo-gestuelles pratiquées par les sourds à travers le monde, sont caractérisées par une forte iconicité, flagrante à tous les niveaux de l'analyse linguistique. Par ailleurs, ces langues semblent présenter entre elles un degré d'invariant bien plus élevé que ce qu'il en est entre langues de modalité audio-phonatoire, les langues vocales (LV) pratiquées par la majorité entendante. Notre contribution explore l'hypothèse selon laquelle ce haut degré de similitude observé entre les LS du monde serait en lien étroit avec leur ancrage commun dans une iconicisation de l'expérience perceptivo-pratique humaine, directement structurante pour ces langues.

Après un exposé succinct de la manière dont la question du variant et de l'invariant est abordée dans la littérature linguistique sur les LS (section 1) et du cadre théorique dans lequel nous appréhendons cette problématique (section 2), nous explicitons la question de recherche précise par laquelle nous l'abordons, et la méthodologie mise en œuvre à cette fin. Il s'agira de l'examen, en transversal d'un échantillon conséquent de LS, de l'un des composants minimaux de ces langues, la configuration manuelle (section 3). Nous verrons que nos premiers résultats (section 4) permettent de corroborer notre hypothèse et d'éclairer ainsi l'apport original et direct des LS à la question plus générale des relations entre perception, langage et langues. Ils montrent aussi l'ampleur de ce qui reste à explorer. 


\section{Langues des signes : variation et invariant}

Les LS, langues naturelles des sourds, sont des langues de modalité visuogestuelle $^{1}$ dont nous noterons une première particularité très remarquable. $\mathrm{Si}$, en effet, les rares recensements existants dénombrent environ 140 LS dans le monde ${ }^{2}$, il ne s'agit là que d'une partie d'entre elles, les LS dites institutionnelles ${ }^{3}$. Il faut y ajouter les probablement très nombreuses LS développées dans l'ontogenèse par un individu sourd isolé en milieu strictement entendant ${ }^{4}$ et, par ailleurs, les diverses LS micro-communautaires pratiquées par de petites communautés de sourds ou, dans le cas de ce qui est désormais souvent désigné par l'expression "village sign languages », des communautés de sourds et d'entendants, et dont le nombre n'est pas non plus établi ${ }^{5}$. Les LS du monde se donnent ainsi à voir, en synchronie, à des degrés divers de développement et de communautarisation. Nous revenons plus loin sur cette réalité étonnante.

Ces langues partagent par ailleurs un ensemble de caractéristiques notables au regard des langues de modalité audio-phonatoire, les LV. Elles mobilisent l'ensemble des articulateurs manuels et corporels c'est-à-dire, outre les deux mains, le regard, l'expression faciale, les mouvements du corps et du visage. Cette possibilité de mouvoir en parallèle ces divers articulateurs dans l'espace devant soi rend compte de deux aspects structurants de ces langues. Il s'agit, d'une part, de l'utilisation massive qu'elles font de l'inscription spatiale des entités du discours (par la direction du regard, un pointage manuel et/ou le placement direct des signes dans l'espace) pour la construction et le suivi des références comme pour l'expression des relations sémantico-syntaxiques. Ces langues, d'autre part, exploitent fortement la simultanéité : la moindre unité gestuelle s'analyse en effet selon une multilinéarité de paramètres, manuels et corporels, dont chacun est spécialisé dans un certain type d'informations linguistiques. Concernant les mains, les paramètres d'analyse classiquement retenus sont leur configuration (forme de la main), leur orientation, leur emplacement (sur le corps ou dans l'espace devant le signeur) et leur mouvement. Par ailleurs, une autre caractéristique commune majeure est la présence flagrante d'iconicité, massive à tous les niveaux de l'analyse linguistique.

Même si la question n'a été thématisée comme telle que très récemment (v. MeIER et al., 2002; WOLL, 2003), les linguistes des LS ont très tôt fait ce constat de fortes ressemblances structurelles de ces langues entre elles, y compris entre LS non apparentées, ceci les éloignant de la diversité caractérisant les LV. Les LS ne diffèreraient fortement entre elles que par leurs lexiques respectifs. Deux facteurs

\footnotetext{
${ }^{1}$ Selon l'usage en linguistique des LS, le terme «modalité » désigne le canal exploité par les langues.

${ }^{2}$ La base de données l'Ethnologue (https://www.ethnologue.com/subgroups/sign-language) recense actuellement $141 \mathrm{LS}$, sans qu'il soit toujours clair qu'il s'agisse de LS institutionnelles au sens défini ici (note suivante).

${ }^{3}$ Une LS est dite « institutionnelle » lorsqu'elle a été intégrée dans un projet d'enseignement, dans un cadre associatif ou scolaire.

${ }^{4}$ Nous reprécisons ce point plus bas. Voir notamment Goldin-Meadow $(1993,2003)$ sur les « homesigns » et FUSELLIER $(2006,2012)$ sur les « LS émergentes ».

${ }^{5}$ Les travaux sur ces divers types de LS sont récents, l'essentiel des recherches jusqu'à la fin des années 1990 ayant porté sur les LS institutionnelles, occidentales de surcroît.
} 
ont été souvent envisagés pour rendre compte de ce fort taux de ressemblance structurelle : la nature modale de ces langues d'une part, leur jeunesse supposée d'autre part, l'une des plus anciennes, la langue des signes française (LSF) étant généralement considérée comme datant du milieu du $18^{\mathrm{e}}$ siècle ${ }^{6}$. Plus récemment, le haut degré de similitude entre LS a lui-même été mis en question : il tiendrait au faible nombre de LS décrites jusqu'ici, qui, en outre, se trouvent être pour l'essentiel des LS institutionnelles, qui plus est occidentales (v. notamment ZESHAN, 2003, 2008). La description de LS d'autres zones géographiques, notamment les LS villageoises, montrerait une diversité plus importante que celle affirmée jusqu'ici (v. par ex. NYsT, 2007, 2012).

Reste toutefois que, même en incluant ces LS nouvellement décrites, ces langues présentent entre elles un taux de ressemblance nettement plus important que ce qu'il en est pour les LV. Un constat empirique, fait depuis longtemps, va dans ce sens : deux locuteurs sourds de LS différentes (y compris non apparentées) ont une capacité à se comprendre et à établir en un court laps de temps des échanges sophistiqués qui est sans commune mesure avec ce qu'il en est pour deux locuteurs de LV différentes. Comment rendre compte de cela ? C'est là notre problématique : qu'en est-il du degré d'invariant entre LS et, surtout, à quels niveaux se situe-t-il ? L'hypothèse que nous défendons, exposée ci-après, est que l'invariant entre LS, effectivement massif, serait lié à leur ancrage commun dans l'expérience perceptivo-pratique.

\section{Langues des signes, langues de sourds : modèle théorique et hypothèse d'un ancrage perceptivo-pratique structurant}

Les linguistes spécialistes des diverses LS dans le monde sont, pour l'essentiel, d'accord sur les particularités communes énumérées ci-dessus. Il existe toutefois un point de clivage majeur, relatif à l'un des deux principaux types d'unités gestuelles reconnues par tous comme constitutives du discours en LS, le désaccord portant sur le statut, linguistique ou non, de l'un de ces deux types.

Deux grands types d'unités ont en effet été identifiés depuis longtemps dans le discours en LS (v. pour L'ASL ${ }^{7}$, Friedman, 1977 ; KLIMA et BeLLUGI, 1979). Le premier type correspond à ce qui est couramment désigné comme «signes lexicaux » ou « words », signes conventionnels comparables aux lexèmes des LV. Le second recouvre les unités complexes le plus souvent désignées comme des « constructions à classificateurs $»^{8}$. La particularité de ces unités (de même format que les signes lexicaux) tient au fait que, bien qu'elles soient composées d'éléments paramétriques manuels de même nature que ceux qui composent les signes conventionnels - où ils sont considérés comme ayant un statut phonémique - chacun de ces composants s'avère porteur de sens, et ce de manière incontestablement

\footnotetext{
${ }^{6}$ L'argument invoqué est celui du taux de similarité important pareillement observé entre les langues créoles, qui partageraient avec les LS le fait d'être « jeunes ».

${ }^{7}$ L'American Sign Language, la LS américaine, dont l'étude a débuté il y a maintenant 57 ans (STOKOE, 1960).

${ }^{8}$ En raison même des désaccords théoriques et descriptifs relatifs à ces unités (v. infra), les termes utilisés sont multiples (voir pour une revue EMMOREY, 2003 et, par ailleurs, GARCIA, 2010 ; GARCIA \& SALLANDRE, 2014).
} 
iconique. La perspective la plus générale dans la littérature (même si elle est controversée) assimile ainsi les configurations manuelles de ces constructions hautement iconiques à des "classificateurs", compris comme référant à des " classes » de référents stables (voir à ce sujet EMMOREY, 2003). Reste que les unités de ce second type sont, dans la majorité des descriptions linguistiques actuelles des LS, jugées comme seulement périphériques et que leur « grammaticalité » est interrogée, les signes lexicaux étant posés comme constituant le cœur des LS, leurs seules unités proprement « linguistiques ».

Une autre approche, celle que nous défendons, a été développée depuis plus de trente ans pour la langue des signes française, la LSF. Fondée sur l'analyse de corpus longs de discours appréhendés en termes fonctionnels (CUXAC, 1985, 1996, 2000, 2013 ; CuXaC et Sallandre, 2007 ; CuXaC et Antinoro Pizzuto, 2010 ; GARCiA et SALLANDRE, 2014), elle a pris récemment le nom de "modèle sémiologique ». Cognitiviste, énonciativiste et fonctionnelle, cette approche fonde en effet la description des langues humaines sur une sémiologie préalable du canal. Elle a permis de mettre en évidence que, à la différence des LV, les LS disposent de deux manières de dire, qui mettent en œuvre deux modes de production du sens. Les unités non conventionnelles y sont décrites comme relevant de structures dites « de transfert » et considérées comme occupant une place centrale, tant en ce qui concerne leur rôle fonctionnel en synchronie que pour ce qui regarde leur rôle dans la phylogenèse et l'évolution diachronique des LS. Le modèle identifie trois principaux types de transferts: les transferts de taille et de forme (TTF), qui permettent de donner à voir la forme et/ou la taille d'une entité ; les transferts de situation (TS), qui montrent le déplacement d'un actant (main dominante) par rapport à un locatif stable (typiquement la main dominée), la scène étant figurée comme vue globalement, à distance ; les transferts de personne (TP), dans lesquels le locuteur devient littéralement l'entité dont il parle et dont il donne ainsi à voir, comme en plan rapproché, les actions effectuées ou subies, ou les discours qu'il tient. Notons que ces structures de transfert rendent compte, de manière unifiée, à la fois des « constructions à classificateurs » de la littérature ( \pm nos TTF et TS) mais aussi d'un autre ensemble de constructions, traitées à part dans le champ, et traditionnellement décrites comme des " prises de rôles » ( \pm nos TP).

Ces structures permettent de produire des unités gestuelles non conventionnalisées, les « unités de transfert» (UT), unités compositionnelles, c'està-dire des unités dont le sens global, à valeur spécifiante, se résume à cette valeur compositionnelle. Les structures de transfert s'ancrent dans le potentiel figuratif propre au canal: elles sont considérées comme résultant d'une structuration linguistique de l'iconicité, comme des structures de l'iconicité. Elles attestent une manière de dire sans équivalent dans les LV, un dire qui donne en même temps à voir, et partagent une même caractéristique formelle qui est qu'elles s'accompagnent d'une rupture du regard sur l'interlocuteur. Il faut y insister, les transferts relèvent bien du verbal: ce sont des structures, contraintes et contraignantes, actualisant en nombre illimité des unités elles-mêmes composées d'éléments entrant dans des paradigmes. Dans cette logique du modèle sémiologique, soulignons-le, la configuration constitutive d'une unité de transfert est appréhendée non pas comme renvoyant à une classe de référents mais comme 
figurant une forme, qu'elle donne à voir comme telle (elle est à ce titre appelée " proforme »), et associée à une valeur de sens d'un haut degré de généricité (par exemple 'forme plate', 'forme tubulaire', etc.).

Les UT se combinent et s'entrelacent en discours avec l'autre grand type d'unités que sont les unités de type lexématique (UL) - les signes conventionnels de la littérature - plus proches, elles, de ce qui existe dans les LV. Ne visant qu'à dire, sans montrer, ces UL sont des unités gestuelles (principalement manuelles) à valeur générique et de sens global conventionalisé. Relevant de la modalité classique du dire, dont le mode de production du sens est la convention (comme il en est des lexèmes des LV), elles sont considérées, à rebours de ce qu'il en est dans le champ, comme seulement périphériques (et les plus susceptibles à ce titre de subir l'influence de la LV environnante). Ces UL sont mobilisées dans un autre type de structures (« structures standard »), ces structures qui, notamment, utilisent l'espace de manière pertinente pour la construction des relations sémantico-syntaxiques (v. section 1). Deux points encore sont importants. Tout d'abord, comme ceci a d'ailleurs été observé par de nombreux auteurs pour diverses LS, les composants paramétriques des UL s'avèrent, le plus souvent et de manière très régulière, porteurs de sens (des morphèmes, donc, et non des phonèmes). Ensuite, pour une partie très importante d'entre eux, ces composants forme-sens des UL sont communs à ceux des UT. Ajoutons enfin que, en discours, les trois types d'UT peuvent se combiner soit entre eux, soit avec une UL, soit encore avec un pointage.

Sur ces bases descriptives, la force de ce modèle est sa portée explicative. Il propose en effet que toutes les LS ont émergé (et émergent ${ }^{9}$ ) à partir d'un même scénario initial, lié à la nature même de la surdité et à la situation des sourds dans un monde majoritairement entendant. Cette hypothèse d'une sémiogenèse commune est corroborée par l'analyse des " homesigns », familiolectes gestuels développés par des enfants sourds isolés en milieu entendant (GOLDIN-MEADOW, 1993, 2003) et la description de LS dites " émergentes » développées dans l'ontogenèse par des adultes sourds isolés au Brésil, sans contact avec la LS institutionnelle (FUSELLIERSoUZA, 2006, 2012). Ces études ont en effet mis en évidence l'existence, dans ces LS créées par des sourds et développées sur l'empan d'une vie, de ces mêmes structures de transfert. Et celles-ci y coexistent, comme dans les LS macrocommunautaires institutionnalisées, avec l'autre type de structures, celles qui manipulent des unités lexicales, conventionnelles. Or, la surdité n'est pas héréditaire : elle est liée à un gène récessif, ceci signifiant que $95 \%$ des enfants sourds naissent de parents entendants, c'est-à-dire dans un environnement linguistique auquel ils n'ont pas d'accès naturel. Il $\mathrm{y}$ a dès lors toute raison de penser que la situation d'un sourd isolé en milieu entendant et sans lien avec une LS institutionnelle - cette situation qui voit émerger ces homesigns et LS individuelles - a dû se produire (et continue de se produire), à l'identique, de tout temps et en tous lieux.

Le scénario de la sémiogenèse qui est proposé - une sémiogenèse commune, donc, à toutes les LS - s'ancre dans ce qui caractérise cette situation réitérée. Il se

\footnotetext{
${ }^{9} \mathrm{C}$ 'est ce qu'attestent les très nombreux homesigns et LS émergentes observables à travers le monde
} (voir section 1 et infra). 
dit dans les termes suivants (v. CUXAC, 2000). Cet individu sourd isolé en milieu entendant est un être de langage et, à ce titre, il est mû par un désir de communiquer. N'ayant pas accès à la LV environnante, il n'a d'autre choix que de recourir au seul canal disponible, son corps, c'est-à-dire à une mise en geste de ce qu'il a précatégorisé du réel. Ce canal étant celui-là même par lequel s'opère, en bonne partie, l'expérience perceptive et praxique du monde, et présentant un fort potentiel figuratif (affordance), cette mise en geste ne peut qu'être iconique (en d'autres termes : pourquoi ne le serait-elle pas ?). Les toutes premières créations gestuelles s'ancreraient ainsi dans une iconicisation de l'expérience perceptivo-pratique, expérience qui est elle-même à l'origine de la formation des catégorisations conceptuelles (images mentales). Si (et seulement si) l'entourage accueille et relaie ces créations, encourageant ainsi l'interaction, le système se développe. L'observation des homesigns et LS émergentes étaye alors l'hypothèse suivante.

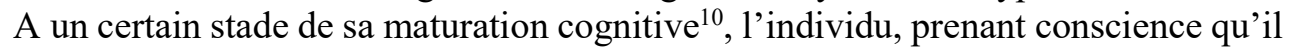
doit se faire comprendre et constatant que plus ses productions sont iconiques, mieux il se fait comprendre, prendrait le parti, cette fois-ci délibéré, de faire systématiquement plus ressemblant encore, pour l'autre. C'est sur les bases de cette intention répétée de "faire iconique » que se serait progressivement opérée une structuration de l'iconicité, traduite en structures de transfert, coexistant avec la stabilisation d'unités gestuelles de valeur générique. Dès lors, c'est parce que le canal visuo-gestuel présente une affinité spécifique avec l'iconique et qu'il se trouve être le seul canal exploitable par une personne qui n'entend pas, que les LS, langues de sourds, auraient exploité maximalement le potentiel langagier de l'iconicité (là où la communication entendante, pouvant faire fond sur deux canaux, répartit entre le vocal et la gestualité dite co-verbale les deux sémiologies respectives du dire et du montrer - les illustrateurs de la gestualité co-verbale, non développés en structures linguistiques) ${ }^{11}$.

Cette conception sous-tend l'hypothèse que fait le modèle sémiologique d'un large tronc commun cognitif et structurel entre toutes les LS du monde (des LS émergentes aux LS macro-communautaires), et d'autant plus large que les unités de transfert sont prises en compte comme proprement linguistiques. Ce tronc commun cognitif et structurel, qui permet de rendre compte des compétences exolingues exceptionnelles des sourds évoquées plus haut ${ }^{12}$, serait ancré dans l'imagerie mentale humaine issue de l'interaction perceptive et praxique avec le monde et s'exprimerait en langue par le processus d'iconicisation initiale (toujours en œuvre dans la néologie) et par les structures de transfert. Dans cette perspective, il n'y a pas de solution de continuité entre les LS émergentes et les LS institutionnelles et, loin d'être des « langues jeunes », ces dernières résultent au contraire assurément d'une histoire bien plus ancienne ${ }^{13}$.

\footnotetext{
${ }^{10}$ Correspondant à celui où se met en place une théorie de l'esprit (voir entre autres CoURTIN, 1998).

${ }^{11}$ Pour les fondements épistémologiques et théoriques de ce modèle et pour le détail de la description, qu'il est bien sûr impossible de développer davantage ici, nous renvoyons le lecteur aux références indiquées plus haut.

12 Dans les faits, la simple observation permet de voir que c'est bien à ces structures de transfert que recourent les sourds de LS différentes lorsqu'ils se rencontrent.

${ }^{13}$ Nous dirons, a minima, que la sémiogenèse des LS se présente différemment de celle des LV.
} 
Les structures de transfert ont récemment fait l'objet d'études comparatives entre plusieurs LS (SALLANDRE, 2014 ; SALLANDRE et al., 2016), dont certaines non apparentées, comme la DGS, la LS allemande. Ces études corroborent fortement l'hypothèse d'un noyau structurel commun constitué des structures de transfert. L'invariant se situe-t-il cependant à ce seul niveau? Ne pourrions-nous pas interroger la présence d'un invariant en amont de ces structures? En d'autres termes, ne se situerait-il pas dès le niveau des composants minimaux constitutifs des unités des LS, composants forme-sens qui, répétons-le, sont en bonne partie communs aux UL et aux UT ? C'est l'hypothèse que notre étude souhaite examiner : leur ancrage dans l'expérience perceptivo-pratique ferait de ces composants des primitives gestuelles iconiques, ancrées cognitivement. Ces composants minimaux, de nature morphémique, constitueraient le premier socle invariant commun aux langues particulières que sont les LS. Selon ARNHEIM (1969), la formation de nos concepts s'ancre en effet dans l'expérience perceptivo-praxique : «La formation des concepts commence par la perception de la forme.» (1969: 35). Et, point important, selon cet auteur, la formation des concepts serait conditionnée non seulement par la perception des formes mais aussi, les deux allant de pair, par leur manipulation : « Les êtres humains [...] explorent et appréhendent par l'action et la manipulation plutôt que par la contemplation. » $(1969: 7)^{14}$.

Dans une telle perspective, on le voit, les LS, langues visuelles et iconiques, apparaissent comme des objets d'étude particulièrement précieux : si minoritaires soient-elles, elles s'appréhendent comme des «fenêtres ouvertes » sur la cognition humaine, particulièrement à même de nous éclairer sur les liens entre langage et perception.

\section{Méthodologie}

Pour évaluer notre hypothèse d'un invariant trans-LS consistant dès le niveau des composants constitutifs des unités gestuelles, nous interrogeons les composants minimaux des unités de plusieurs LS, dont certaines ne sont pas génétiquement liées et qui sont par ailleurs pratiquées dans des zones géographiques et culturelles diversifiées. Nous attachons une attention particulière aux LS non reconnues institutionnellement et utilisées par des communautés restreintes - les LS dites villageoises. Notre objectif est de déterminer dans quelle mesure les composants forme-sens des unités gestuelles sont similaires en transversal des LS étudiées. Pour ce faire, nous procédons en deux temps.

Précisons cependant que, parmi les quatre composants manuels, nous avons fait le choix de nous centrer sur la configuration. Ceci, d'une part, en raison de l'abondante littérature existant sur ce composant, d'autre part, parce que, plus stable par nature, il est relativement plus facile à appréhender que l'emplacement ou, surtout, le mouvement. En outre, ce composant nous semble se prêter plus spécifiquement à la représentation gestuelle des percepts de formes que, pour certains d'entre eux, ils peuvent même directement incarner, nous y reviendrons.

${ }^{14}$ Sur ce point, notons que les LS pratiquées par les sourds-aveugles, dont la structuration est, elle, tributaire de la modalité haptique, auraient beaucoup à apporter (voir notamment, à ce sujet, SCHWARTZ, 2009). 
La première étape de notre méthodologie a consisté à identifier et analyser les inventaires de configurations disponibles pour les LS décrites à ce jour, inventaires ne devant pas se limiter à une simple liste des configurations utilisées dans une LS donnée, mais, dans la mesure du possible, à des listes de configurations régulièrement associées à une ou plusieurs valeurs de sens. A cet égard, nous nous sommes heurtées à diverses difficultés.

En accord avec la prévalence dans la littérature de l'affirmation du statut phonologique des composants paramétriques des unités conventionnelles, les inventaires que nous avons pu trouver se sont avérés être principalement des inventaires de configurations considérées comme de simples formes, dépourvues de sens. Même s'agissant de ces inventaires de configurations strictement phonologiques, un premier constat est leur caractère non exhaustif (ce point avait déjà été souligné par BOUTORA, 2008) ${ }^{15}$. Mais les rares inventaires de configurations directement appréhendées comme unités morphosémantiques se sont eux aussi avérés bien souvent incomplets, y compris pour la LS la plus étudiée, l'ASL, ces inventaires étant fournis dans un but simplement illustratif, sans prétention à l'exhaustivité, et pour les seules unités dites «non conventionnelles » ( « constructions à classificateurs »). Enfin, les modes de représentation graphique des configurations diffèrent selon les inventaires, chaque auteur utilisant une notation qui lui est propre (captures d'écran d'un corpus vidéo, dessins ou codes divers). Une phase d'interprétation, de sélection et d'homogénéisation a donc été nécessaire, en vue de ne retenir que des inventaires comparables. Les inventaires retenus au final concernent les LS suivantes : la LSF (CUXAC, 2000), la LS turque (TID) (KUBUŞ, 2008), la LS néerlandaise (NGT) (ZWITSERLOOD, 2012), la LS britannique (BSL) (BRENNAN, 1990), la LS inuit (IUR) (SCHUIT, 2014) et la LS de Shanghaï (SSL) (NI, 2015) pour les LS institutionnelles, et trois LS du centre du Brésil (FUSELLIER-SOUZA, 2004) pour les LS micro-communautaires.

La deuxième étape est la constitution de notre propre inventaire de configurations morphémiques à partir de LS non encore décrites et constituant un cas de figure inédit parmi les types de LS non institutionnelles. Ces LS, pratiquées dans une région rurale, l'île de Marajó, au nord-est du Brésil, sont en effet actuellement en cours de communautarisation. Les sourds de cette région vivaient chacun isolément dans sa famille entendante jusqu'en 2010. A cette date, l'annexe locale de l'Université fédérale de l'État de Parà (UFPA) a mis en place des rencontres entre les sourds de la capitale de l'île (Soure), favorisant ainsi une amorce de communautarisation des homesigns pratiqués auparavant dans chaque famille où vivait au moins une personne sourde. En appui à ce que nous avons indiqué plus haut (section 1.) et à notre scénario de la sémiogenèse, les LS développées dans des régions rurales ne sont pas des exceptions dans le monde. Au contraire, il semblerait qu'il s'agisse de la norme dès lors qu'un individu sourd naît dans une région reculée, sans accès à l'éducation. Ce qui fait de l'île de Marajó une situation rare est qu'elle offre la possibilité d'assister in vivo à la communautarisation des LS idiosyncrasiques de signeurs sourds jusque-là isolés.

\footnotetext{
${ }^{15}$ Notons simplement ici, au passage, que ce constat contribue à fragiliser l'affirmation du caractère phonologique de ces composants.
} 
Sur le terrain, nous avons réalisé un travail de recueil de données à partir des productions de six locutrices sourdes, obtenues au moyen de stimuli imagés ou d'enregistrements de conversations spontanées ${ }^{16}$. Sur les vingt heures du corpus vidéo ainsi constitué, nous avons sélectionné certains passages (dix minutes environ) issus de types de discours différents, élicités ou spontanés. Ces passages ont ensuite été annotés sous ELAN ${ }^{17}$ suivant un schéma d'annotation ciblant particulièrement le relevé, l'analyse et la comptabilisation des configurations utilisées par les locutrices. A l'arrivée, cette étape permet la constitution d'un inventaire des configurations morphémiques des (de la ?) LS du Marajó à même d'être comparé aux sept autres inventaires sélectionnés.

\section{Analyses et discussion}

Premier résultat important, cinq configurations forme-sens, figurées dans le Tableau 1 ci-dessous, se retrouvent à l'identique dans toutes les LS investiguées. Ces configurations ont été décrites dans la littérature comme étant physiologiquement les plus simples à produire (JOHNSTON, 1989; BATTISON, 1978), ce qui pourrait certes expliquer leur présence dans chacune de ces LS. Le point notable toutefois est leur association systématique aux mêmes valeurs de sens en transversal des LS examinées.

\footnotetext{
${ }^{16}$ Pour le détail de la méthodologie, voir MARTINOD (2013).

${ }^{17}$ Logiciel d'annotation de corpus vidéo élaboré par le Max Planck Institute for Psycholinguistics, The Language Archive, Nijmegen, The Netherlands (http://tla.mpi.nl/tools/tla-tools/elan/). Voir Crasborn, O., Sloetjes, H. (2008). ElAN est le logiciel d'annotation couramment employé en linguistique des LS.
} 


\begin{tabular}{|c|c|c|c|c|c|}
\hline & सी & A & ลิ & d & 21 \\
\hline $\begin{array}{l}\text { Procédé } \\
\text { iconique }\end{array}$ & $\begin{array}{l}\text { Reprise de } \\
\text { forme mince } \\
\text { et allongée. } \\
\text { Reprise de } \\
\text { taille. }\end{array}$ & $\begin{array}{l}\text { Reprise de } \\
\text { forme/surface } \\
\text { plate. } \\
\text { Reprise de } \\
\text { taille. }\end{array}$ & $\begin{array}{l}\text { Reprise de } \\
\text { forme petite } \\
\text { et ronde } \\
\text { bidimension- } \\
\text { nelle. } \\
\text { Reprise de } \\
\text { forme } \\
\text { allongée et } \\
\text { cylindrique (à } \\
\text { deux mains). }\end{array}$ & $\begin{array}{l}\text { Reprise de } \\
\text { forme } \\
\text { allongée dont } \\
\text { les côtés sont } \\
\text { approximati- } \\
\text { vement } \\
\text { parallèles. }\end{array}$ & $\begin{array}{l}\text { Reprise de } \\
\text { forme } \\
\text { tubulaire ou } \\
\text { cylindrique. }\end{array}$ \\
\hline $\begin{array}{l}\text { Valeur } \\
\text { sémantique }\end{array}$ & $\begin{array}{l}\text { Forme mince } \\
\text { et allongée. } \\
\text { Spécificateur } \\
\text { de taille. }\end{array}$ & $\begin{array}{l}\text { Forme / } \\
\text { surface plate. } \\
\text { Spécificateur } \\
\text { de taille. }\end{array}$ & $\begin{array}{l}\text { Forme } \\
\text { bidimension- } \\
\text { nelle petite et } \\
\text { ronde. } \\
\text { Forme } \\
\text { allongée et } \\
\text { cylindrique. }\end{array}$ & $\begin{array}{l}\text { Forme } \\
\text { allongée dont } \\
\text { les côtés sont } \\
\approx \text { parallèles. }\end{array}$ & $\begin{array}{l}\text { Forme } \\
\text { tubulaire ou } \\
\text { cylindrique. }\end{array}$ \\
\hline
\end{tabular}

Tableau 1 : Les configurations forme-sens communes aux LS investiguées

Par ailleurs, sept autres composants forme-sens se retrouvent à l'identique dans plus de la moitié des LS de notre panel, y compris, souvent, des LS non apparentées, comme la NGT et la LSC par exemple. Le fait que cette similarité ne puisse être due à une parenté génétique ou un quelconque contact de langue corrobore notre hypothèse de l'incidence directe d'un semblable ancrage dans l'iconicisation de l'expérience. Plus encore, en-deçà des configurations morphémiques en ellesmêmes, nous observons que les procédés d'iconicisation utilisés par les LS étudiées, en nombre restreint qui plus est, sont eux aussi identiques d'une LS à l'autre. Il s'agit soit d'une simple « reprise de forme », la forme étant représentée directement par la forme de la main (par exemple : renvoie à une forme mince et allongée), soit d'une "saisie de forme », dans laquelle la main prend la forme requise pour la saisie de l'entité que l'on veut signifier (2 reflète ainsi la saisie d'une forme mince et rigide pour signifier une forme mince et rigide), soit encore d'une « reprise de taille $\gg$ ( signifie une entité en en indiquant la taille).

À notre échelle, ce constat conforte non seulement notre hypothèse d'un principe d'iconicisation du réel à partir de primitives cognitives mais il nous mène également vers de possibles primitives de catégorisation. Quoi qu'il en soit, ces trois procédés relèvent bien de stratégies récurrentes au fondement de la mise en geste des percepts. Quant au fait de savoir si l'une ou l'autre de ces stratégies serait privilégiée, il est intéressant de constater que les cinq composants forme-sens communs aux huit LS investiguées ainsi que les sept autres fortement similaires en transversal de notre panel de LS sont majoritairement des configurations de reprise de forme ${ }^{18}$. Ceci va à l'encontre de l'hypothèse avancée dans la littérature selon

\footnotetext{
${ }^{18}$ Précisons qu'une configuration donnée peut être à la fois utilisée comme reprise de forme et comme saisie de forme dans une LS. Ainsi la configuration en LSF correspond-elle à au moins deux formesens (deux morphèmes), l'une signifiant une « forme sphérique » (reprise), l'autre une « forme mince
} 
laquelle les configurations de reprise de forme seraient celles qui présenteraient le plus de variation entre LS (JOHNSTON et SCHEMBRI, 1999; PFAU, 2010). Notons que selon VELUPILLAI (2012), cette hypothèse n'a jamais été vérifiée sérieusement jusqu'à présent. Notre travail de comparaison ne va en tout cas pas dans ce sens.

Nous avons, pour la suite de notre examen, adopté une démarche onomasiologique, c'est-à-dire que, en partant de valeurs sémantiques génériques, nous nous sommes demandé quelle(s) solution(s) étai(en)t adoptée(s) dans chaque LS pour exprimer tel ou tel concept de forme (tel ou tel percept). Cette démarche nous a permis de remarquer certains éléments qui nous auraient échappé par l'observation des formes seules. Pour comprendre notre approche, il faut distinguer trois domaines : ceux de la cognition, du langage et de la langue. Dans la perspective qui est la nôtre et en accord avec ARNHEIM, les percepts sont d'authentiques concepts, qui relèvent, donc, du domaine de la cognition. Ainsi, le percept/concept d'une forme rectangulaire plate, dans le domaine cognitif, va donner lieu à une représentation gestuelle de ce concept. En d'autres termes, les primitives cognitives vont être à leur tour instanciées par des primitives gestuelles. Mais pour un même concept, il est envisageable qu'il y ait plusieurs représentations gestuelles possibles. $\mathrm{Au}$ concept de «forme à double saillance latérale», par exemple, peuvent correspondre quatre représentations gestuelles ( passe comme si chaque LS puisait dans cet ensemble de possibles. Le concept de " forme rectangulaire plate » est représenté par en LSF, LS britannique, LS de Shanghai, LS néerlandaise et dans les LS émergentes du centre du Brésil et par $\varphi^{\ell}$ en LSF et LS turque. Dans ce cas précis, semble être la solution la plus productive, probablement en raison d'une articulation relativement plus aisée que celle de g et, peut-être, d'une saillance perceptive supérieure.

Par ailleurs, le choix opéré par la langue peut également concerner le procédé iconique mis en œuvre dans la représentation gestuelle : le concept de " forme tubulaire » pourra être exprimé à la fois par une reprise et une saisie de forme en LSF ( ) là où, en LS turque, il sera exprimé uniquement par une reprise de forme, sans équivalent de saisie de forme (du moins d'après l'inventaire disponible).

Enfin, en partant de valeurs de sens - ici des concepts/percepts de forme visuelle, nous observons parfois des «vides » dans certaines LS. Entre autres cas, le concept de « forme rectangulaire plate» ne semble trouver aucune configuration dédiée en LS inuit, que ce soit en ayant recours à une reprise ou à une saisie de forme. Plusieurs hypothèses peuvent expliquer cette absence. Premièrement, la LS en question n'a peut-être aucun moyen d'exprimer ces concepts de forme car elle n'en a pas besoin, ces derniers n'étant peut-être pas fortement présents dans l'environnement immédiat des locuteurs. Une deuxième hypothèse serait que la LS aurait simplement recours à un procédé d'iconicisation plus rare, différent des trois précédemment cités (reprise de forme, saisie de forme, ou reprise de taille). Enfin, la troisième hypothèse reviendrait à interroger les modalités d'élaboration des inventaires que nous utilisons dans notre analyse, et leur fiabilité. En effet, nous travaillons sur des données de seconde main et en cela, nous ne pouvons pas être

et allongée » (saisie). C'est bien de « reprise de forme » dont il s'est agi majoritairement dans les inventaires examinés, et non de « saisie ». 
garantes des différentes étapes de la constitution des inventaires récoltés, ni des présupposés de recherche qui ont guidé leurs auteurs. Notre cadre théorique nous amène en outre à utiliser une terminologie spécifique qui spécifie les procédés d'iconicisation. Or, la plupart des inventaires examinés ne donnent généralement pas autant de détails. Il est donc peu aisé de comparer « ce qui est comparable ».

La première hypothèse, celle de la simple absence d'expression d'un concept donné dans telle LS, rejoint, toutes proportions gardées, le cas de la langue (vocale) dani, utilisée en Papouasie occidentale (exemple cité par Rosch, 1973) : cette langue n'aurait pas de lexème pour les formes géométriques. Pour SEGALL, CAMPBELL et HERSKOVITZ (1966), ceci s'expliquerait par le fait que l'environnement quotidien de la région est exempt de formes en trois dimensions dont les contours seraient réguliers, ce qui aurait pu amener le besoin d'utiliser des lexèmes pour référer à ces formes. Pour le cas de la LS inuit, un tel type d'hypothèse semble envisageable si l'on pense àl'habitat typique de cette population. Concernant la deuxième hypothèse, alternative à la précédente, il n'est pas exclu que les locuteurs de cette LS inuit aient recours à une stratégie autre, notamment celle consistant à représenter le contour de la forme rectangulaire plate par traçage dans l'espace de signation. Cependant, pour confirmer ou infirmer cette deuxième hypothèse (mais également la troisième), il nous faudrait analyser un corpus de cette LS.

Ainsi, si nos premiers résultats sont substantiels et vont dans le sens de notre hypothèse de départ, ils doivent évidemment être complétés. Nous avons insisté sur les limites liées aux inventaires analysés. A titre d'exemple, nous avons été confrontées au cas de configurations absentes de l'inventaire élaboré pour telle LS, alors même que nous avions pu par ailleurs en vérifier l'existence dans le dictionnaire élaboré pour cette langue. C'est le cas notamment de en LS turque, qui était absent de l'inventaire mais apparaissait pourtant dans certains signes lexicaux de la LS turque comme LUNETTES, ce qui est par ailleurs peu surprenant au vu de la forme de cet objet et du concept de forme évident auquel il est lié (« forme ronde plate »).

Nous nous interrogeons par ailleurs sur l'utilisation préférentielle de telle ou telle représentation gestuelle. Existe-t-il de meilleurs candidats pour certains percepts et, si tel est le cas, pour quelles raisons ? Nous avons évoqué les critères de saillance perceptive et de facilité articulatoire mais dans quelle mesure est-ce pertinent et y aurait-il d'autres critères en jeu ? Nous n'avons pas évoqué la question des tabous culturels liés à l'utilisation de certaines configurations (pensons à encore dans certaines cultures) mais ceci joue assurément un rôle dans les choix des locuteurs et, partant, dans les inventaires retenus par telle ou telle LS.

Enfin, nous avons observé que, en LSF, pour l'expression du concept de « forme rectangulaire plate $»$, deux configurations semblaient possibles : et ty examen de leur utilisation dans la langue, il semblerait qu'elles soient liées à une nuance sémantique : est en effet utilisée lorsque la forme est statique (référant à un tableau par exemple), tandis que l'est pour une forme mobile, comme dans FENETRE. Est-ce toujours le cas lorsque deux candidats gestuels sont retenus dans une langue ? Le critère de «possibilité de mouvement »a-t-il une incidence sur 
d'autres percepts de formes? Par ailleurs, ce critère vaut-il pour toutes les LS ou seulement pour certaines d'entre elles? Sur ce point, si l'on revient au cas des représentations gestuelles multiples évoqué plus haut ( 1,1, , the en tant que reprise d'une « forme à double saillance latérale »), il semblerait que le parallélisme des deux saillances de la forme en question ne revête pas la même importance selon les LS. Ainsi, cette différence semble être très marquée pour la LS britannique ou la LS turque - on utilisera alors $1 /$ voire th pour les saillances non nécessairement parallèles mais obligatoirement pour les saillances parallèles - mais l'est nettement moins pour la LSF, par exemple, où ces configurations semblent davantage interchangeables.

\section{Conclusion}

Nos premiers résultats suggèrent fortement que la part d'invariant dans les LS s'étend en-deçà des structures de transfert. Cet invariant existe déjà au niveau même des composants minimaux dont on peut ainsi faire l'hypothèse qu'une partie au moins d'entre eux sont des icônes de primitives cognitives façonnées par l'expérience perceptivo-pratique humaine. Cette expérience donnerait lieu à différents procédés d'iconicisation des percepts, en nombre réduit, ainsi qu'à un noyau substantiel de composants forme-sens communs, exprimés par la configuration manuelle. Notre analyse a certes permis de dégager un certain nombre de similitudes entre LS mais nous devons encore affiner nos résultats. En effet, les critères retenus dans la catégorisation des primitives cognitives ancrées dans l'expérience, la variation de ces critères ainsi que leur importance relative restent encore à préciser.

Notre cadre théorique prend d'emblée en compte le rôle primordial de l'expérience humaine du monde pour la structuration de la pensée et du langage. Il permet d'aborder l'examen des inventaires de configurations morphémiques d'un point de vue original par rapport à ceux qui prévalent dans les analyses habituelles de ce composant. Ces dernières, nous l'avons souligné, associent généralement telle configuration de ces constructions iconiques à une classe de référents sans interroger le rôle des percepts/concepts de forme dans ce choix.

Il nous reste cependant à chercher les raisons de la présence de certains composants forme-sens en transversal des LS : pourquoi ceux-ci plutôt que d'autres ? Ces percepts semblent à même de nous éclairer sur la compréhension des relations entre perception, langage et langue. Nous souhaitons par la suite étendre notre analyse à d'autres LS et, à terme, cette investigation sera à mener sur les autres composants manuels, ces derniers participant également, bien sûr, au processus d'iconicisation de l'expérience.

\section{BIBLIOGRAPHIE}

ARNHEIM Rudolf (1969), Visual thinking, University of California Press, Berkeley. BATTISON Robin (1978), Lexical borrowing in American sign language. Silver Spring, MD: Linstock Press, Burtonsville, Maryland.

BOUTORA Leïla (2008), Fondements historiques et implications théoriques d'une phonologie des langues des signes - Etude de la perception catégorielle des 
configurations manuelles en LSF et réflexion sur la transcription des langues des signes, Thèse de doctorat en Sciences du Langage, Université Paris 8.

BRENNAN Mary (1990), Word formation in BSL, Stockholm: Department of Linguistics, Stockholm University.

CourTIN Cyril (1998), Surdité, langue des signes et développement cognitif. Thèse de doctorat, Université Paris 5.

CRASBORN Onno, \& SLOETJES Han (2008), Enhanced ElAN functionality for sign language corpora. In: Nicoletta Calzolari, Khalid Choukri, Bente Maegaard, Joseph Mariani, Jan Odjik, Stelios Piperidis and Daniel Tapias (eds.), Sixth International Conference on Language Resources and Evaluation (LREC 2008)/3rd Workshop on the Representation and Processing of Sign Languages: Construction and Exploitation of Sign Language Corpora, 39-43. eds. ELDA, Parijs.

CUXAC Christian (1985). Esquisse d'une typologie des langues des signes. In: CuXAC Christian (éd.), Autour de la langue des signes, Journées d'Études 10, UFR de linguistique générale et appliquée, Université René Descartes, Paris, 35-60.

CuXAC Christian (1996), Fonctions et structures de l'iconicité des langues des signes. Analyse descriptive d'un idiolecte parisien de la langue des signes française, Thèse d'État en Sciences du Langage, Université Paris 5.

CUXAC Christian (2000), La langue des signes française (LSF): les voies de l'iconicité, Faits de langue 15-16. Ophrys, Paris.

CuXAC Christian, ANTINORO PIZZuTO, Elena (2010), Émergence, norme et variation dans les langues des signes : vers une redéfinition conceptuelle, Langage et société 131(1), 37-53, Paris.

CUXAC Christian \& SALLANDRE Marie-Anne (2007), Iconicity and arbitrariness in French Sign Language: Highly Iconic Structures, degenerated iconicity and diagrammatic iconicity. Empirical approaches to language typology 36, 13-33. Berlin: Mouton de Gruyter.

CuXAC Christian (2013), Langues des signes: une modélisation sémiologique. La nouvelle revue de l'adaptation et de la scolarisation (4)65-80. Numéro coordonné par Andrea Benvenuto et Didier Séguillon, Éditions de l'INS HEA, Suresnes.

EMMOREY Karen (2003), Perspectives on classifier constructions in sign languages. Psychology Press, Mahwah: Lawrence Erlbaum Assoc.

FrIEDMAN, Lynn Ann (1977). On the other hand: New perspectives on American Sign Language. New York: Academic Press.

FUSELLIER-SOUZA Ivani (2004), Sémiogenèse des langues des signes: étude de langues des signes primaires (LSP) pratiquées par des sourds brésiliens, Thèse de doctorat en Sciences du Langage, Université Paris 8.

FUSELLIER-SOUZA Ivani (2006), Emergence and Development of Signed Languages: From a Semiogenetic Point of View, Sign Language Studies 7(1), 30-56. Gallaudet University Press.

FUSELLIER-SOUZA Ivani (2012), Multiple Perspectives on the Emergence and Development of Human Language: Bernard Comrie, Clive Perdue and Dan Slobin. In: WATOREK Marzena, BenAzzo Sandra, HickManN Maya (éd.), 
Comparative perspectives on language acquisition : a tribute to Clive Perdue, 223-244, Bristol.

GARCIA Brigitte (2010), Sourds, surdité, langue(s) des signes et épistémologie des sciences du langage : problématiques de la scripturisation et modélisation des bas niveaux en Langue des Signes Française (LSF), Thèse d'habilitation à diriger les recherches en Sciences du Langage, Université Paris 8.

GARCIA, Brigitte \& SALLANDRE, Marie-Anne (2014), Reference resolution in French Sign Language. In Patricia Cabredo Hofherr and Anne Zribi-Hertz (eds.), Crosslinguistic studies on Noun Phrase structure and reference [Syntax and semantics series 39], 316-364. Leiden, Brill.

GOLDIN-MEADOW Susan (2005), The resilience of language: What gesture creation in deaf children can tell us about how all children learn language, Psychology Press, New-York.

GOLDIN-MEADOW Susan (1993), When does gesture become language? A study of gesture used as a primary communication system by deaf children of hearing parents. In K. R. GIBSON \& T. INGOLD (eds.), Tools, language and cognition in human evolution. New York, Cambridge University Press, pp. 63-85.

JOHNSTON Trevor Alexander (1989), Auslan: The sign language of the Australian deaf community, University of Sydney, Sydney.

JOHNSTON Trevor Alexander \& SCHEMBRI Adam (1999), On defining lexeme in a signed language. Sign language \& linguistics 2(2), 115-185. Gallaudet University Press.

Klima Edward \& Bellugi Ursula (1979), The Signs of Language, Cambridge, MA: Harvard University Press.

KuBUŞ Okan (2008), An analysis of Turkish Sign Language (TID) phonology and morphology. Doctoral thesis, Middle east technical university. Ankara, Turquie.

MARTINOD Emmanuella (2013), Les LS pratiquées par des sourds isolés de Marajó, Mémoire de Master 2 de Sciences du langage, Université Paris 8 - ENS-Ulm.

MARTINOD Emmanuella, GARCIA Brigitte, FUSELliER Ivani (en révision), Morphemic handshapes in the sign languages on Marajo Island (Brazil) in a typological perspective. In: Emerging Sign Languages in South America, Berlin: Mouton de Gruyter.

MEIER Richard P. \& CORMIER Kearsy (2002), Modality and structure in signed and spoken languages, Cambridge University Press.

Ni Lan (2015), Study of Verbs in Chinese Sign Language, Shanghai University Press, Shanghai.

NYST Victoria (2007), a descriptive analysis of Adamorobe sign language (Ghana). Doctoral thesis, Netherlands Graduate School of Linguistics.

Nyst Victoria (2012), Shared sign languages. In: Sign languages, An International Handbook, 552-574. Berlin: Mouton de Gruyter.

PFAU Roland (2010), Handwaving \& headshaking? On the linguistic structure of sign languages. In J. Martí i Castell \& J. M. Mestres i Serra (eds.). Les llengües de signes com a llengües minoritàries: Perspectives lingüistiques, socials $i$ polítiques: actes del seminari del CUMIPB-CEL 2008 (59-84). Barcelona: Institut d'Estudis Catalans. 
Rosch Eleanor Heider (1973), Natural categories, Cognitive psychology 4(3), p. 328-350. Cambridge, Massachusetts.

SALlANDRE Marie-Anne (2014), Compositionnalité des unités sémantiques en langues des signes. Perspective typologique et développementale, Thèse d'habilitation à diriger des recherches en Sciences du Langage, Université Paris 8.

SALlANDRE Marie-Anne, Di RENZO Alessio \& GAVRILESCU Robert (2016), Various types of personal transfers (constructed actions) in seven sign languages. Poster, 12th International Conference on Theoretical Issues in Sign Language Research (TISLR12), Australia, 4-7 January.

SCHUIT Joke (2014), Signs of the arctic: Typological aspects of Inuit Sign Language. Amsterdam: Universiteit van Amsterdam, $\mathrm{PhD}$ Dissertation.

Segall, Marshall H., CAMPBell, Donald T., \& HersKovits, Melville J. (1966), The influence of culture on visual perception, Bobbs-Merrill Indianapolis.

SCHWARTZ Sandrine (2009), Stratégies de synchronisation interactionnelle alternance conversationnelle et rétroaction en cours de discours-chez des locuteurs sourds-aveugles pratiquant la Langue des Signes Française tactile, Thèse de doctorat en Sciences du Langage, Université Paris 8.

StokоE, William C. (1960), Sign language structure: An outline of the visual communication systems of the American deaf. Journal of deaf studies and deaf education 10.1, pp. 3-37.

VeluPILlaI Viveka (2012), An Introduction to Linguistic Typology, Amsterdam, John Benjamins Publishing Company.

Woll Bencie (2003), Modality, universality, and the similarities among sign languages: an historical perspective. In: Ann E. Baker, Beppie van den Bogaerde \& Onno Crasborn (eds.), Crosslinguistic perspectives in sign language research: Selected papers from TISLR 2000, 17-31. Hamburg: Signum Press.

ZESHAN Ullrike (2003), Indo-Pakistani Sign Language grammar: a typological outline, Sign Language Studies 3(2), 157-212. Gallaudet University Press.

ZESHAN Ullrike (2008), Roots, leaves and branches - The typology of sign languages. In Quadros, R. M. de (ed.) Sign Languages: Spinning and Unraveling the Past, Present and Future. 45 Papers and 3 Posters from the 9th Theoretical Issues in Sign Language Research Conference. Petrópolis: Editora Azul, 671-695.

ZWITSERLOOD Inge (2003), Classifying hand configurations in Nederlandse Gebarentaal (Sign Language of the Netherlands), Thèse de doctorat, Université d'Utrecht. 